\title{
Skeletal complications of ADT: disease burden and treatment options
}

\author{
Jacques Planas Morin and Juan Morote Robles
}

Therapy based on androgenic deprivation is one of the standard treatments that many prostate cancer patients receive. Moreover, its use is increasing owing to a clear expansion of the indications for this therapy in patients with localized prostate cancer. Despite classically being considered to be well tolerated, androgenic deprivation has adverse effects. Of these, the loss of mineral bone mass is particularly notable and can lead to osteoporosis, as well as an increased risk of bone fracture. Some fractures, such as hip fractures, may have serious consequences. Useful procedures such as bone densitometry can aid in the diagnosis of these conditions. Once diagnosed, decreases in mineral bone mass can be managed by dietary recommendations, general changes in lifestyle or medication. We review the most important randomized controlled trials evaluating different drugs (bisphosphonates, denosumab and toremifene) in the prevention of bone loss and in the reduction in fracture risk in prostate cancer patients treated with androgen-deprivation therapy. Following the applicable recommendations, urologists must carefully monitor the bone health of prostate cancer patients subjected to androgenic deprivation to obtain an early diagnosis and apply the appropriate general and/or therapeutic measures if necessary. Asian Journal of Andrology (2012) 14,670-675; doi:10.1038/aja.2012.70; published online 20 August 2012

Keywords: androgen deprivation therapy; bisphosphonates; bone fracture; bone mass loss; calcium intake; osteoclast; osteoporosis; prostate cancer

\section{INTRODUCTION}

Prostate cancer is one of the most common malignancies with regards to incidence and prevalence. Epidemiological data in the United States estimate an incidence of 155 new cases per 100000 inhabitants in $2010 .{ }^{1}$ In Spain, we estimate that there were 25000 new cases and 6000 deaths in 2008, representing an incidence of 57 new cases and 10 deaths per 100000 inhabitants per year. It is likely that regional/ national differences in incidence are due to the use of different measures by the respective population registers or to different protocols for handling prostate-specific antigen screening. Nonetheless, these data clearly justify the claim that prostate cancer is a major public health problem.

Historically, bones have been a subject of much interest among urologists because malignant prostate cancer cells have a special bone trophism, explaining why bone tissue is the most common metastatic location for this type of tumour. This bone trophism results from anatomical factors, such as the Batson paravertebral plexus, which connects the prostate with the axial skeleton, as well as biological factors, such as parathyroid hormone-related protein and transforming growth factor- $\beta$. These factors establish a feedback loop between prostate cancer cells and the bone microenvironment that facilitates bone metastasis establishment and growth. ${ }^{2}$ Once metastases have appeared, prostate cancer is considered incurable, and the treatment of choice at that time is androgen-deprivation therapy (ADT); chemotherapy is reserved for second-line treatment.
The favourable effect of ADT in patients with prostate cancer has been known for the past 50 years, and ADT is generally considered a well-tolerated treatment. However, ADT carries several side effects that can become troublesome for patients. Adverse effects attributable to androgen loss include sarcopenia or decreased lean body mass, obesity, anaemia, hair loss, erectile dysfunction and insulin resistance. Also, since estrogen in men is primarily derived from testosterone aromatisation, ADT results in low estrogen (greater than $70 \%$ decrease) and subsequent estrogen deficiency-related effects. These effects include gynaecomastia, hot flashes, mental and emotional symptoms, and unfavourable lipid profile changes. Studies also indicate an increased risk of incident diabetes and cardiovascular morbidity for short-term (6 months or less) and long-term ADT.

Sexual hormones play an important role in bone remodelling regulation. Regarding sexual hormones, estrogens have been much more investigated than androgens. We know that when estrogen levels are low or absent, an increase in the number of osteoclasts is observed, followed by an increase in osteoblasts, which lead to an increase in the bone remodelling process. It is not clear whether estrogens play its role directly (through estrogen receptors located at bone cells) or indirectly through different local factors like IL-1, tumour necrosis factor- $\alpha$, IL-6 or prostaglandins. ${ }^{3}$ Since estrogens have a central role in male bone resorption and formation, and the maintenance of bone mass, ADT may also result in adverse skeletal effects. Specifically ADT increases bone turnover and clinical fractures, and decreases bone mineral density (BMD). ${ }^{4}$

Department of Urology, Hospital Vall d'Hebrón, Universitat Autònoma de Barcelona, Barcelona 08035, Spain 
The first study linking ADT with osteoporosis was published in 1997: Daniell $^{5}$ analysed the effect of orchiectomy in 235 patients with prostate cancer and showed that this procedure was associated with a subsequent increase in the risk of osteoporosis and osteoporotic fractures. Also in 1997, another study was published showing similar results in patients treated with luteinizing hormone-releasing hormone analogues. ${ }^{6}$

In patients with non-metastatic prostate cancer, $\mathrm{ADT}$ has been shown to provide clinical benefits in terms of increased survival in two specific areas: when administered with radiotherapy to patients with high-risk localized prostate cancer, and when used as an adjuvant treatment with radical prostatectomy in patients with lymph node disease. Thus, the indications for using ADT have been expanded, and ADT is now used as a neoadjuvant or adjuvant therapy in some patients with non-metastatic tumours at diagnosis. This has resulted in a steady increase in the number of patients treated with ADT in the past few years, especially in patients who would have been previously treated exclusively with radiotherapy. ${ }^{7}$ Many of these patients now have longer disease-free survival rates and even a chance of being cured. However, a population-based study showed that the widespread use of prostate-specific antigen screening in elderly patients resulted in an increase in ADT being prescribed ${ }^{8}$ rather than a decrease, which was the initial hypothesis. As a result, today we are seeing the emergence of complications from long-term ADT that were previously unknown because they were found only in long-term survivors of metastatic prostate cancer. The loss of bone mineral mass is one of the most important complications related to ADT.

During adulthood, bone tissue is subjected to a continuous remodelling process based on coordinated bone formation and resorption, which are mediated by the actions of osteoblasts and osteoclasts, respectively. The function of osteoclasts is regulated by various factors, such as the ligand receptor activator of nuclear factor- $\kappa \mathrm{B}$ (RANK) and osteoprotegerin. ${ }^{9}$ The binding of RANK to its ligand (RANKL) leads to the activation of a signal transduction pathway that mediates the formation and survival of osteoclasts, stimulating their function. Conversely, the binding of osteoprotegerin to RANKL leads to the inhibition of bone resorption mediated by osteoclasts. Thus, $\mathrm{BMD}$ is the result of a complex series of balances at the cellular (osteoblast-osteoclast) and molecular (RANKL-osteoprotegerin) level.

The physiology of the bone remodelling process, mediated by multiple cytokines and hormones, explains why patients receiving ADT may have a decreased bone mineral mass. Oestrogens inhibit bone resorption, whereas androgens promote bone formation. Alteration of the balance between these mechanisms as a result of menopausal status, treatment with aromatase inhibitors in breast cancer patients or with ADT in prostate cancer patients leads to an increased risk of bone loss.

The main objective of the present review is to provide information about the importance of bone loss resulting from ADT, as well as its incidence, prevalence and known risk factors, and to review the scientific knowledge currently available. We also systematically review the state-of-the-art diagnostic and therapeutic manoeuvres (both pharmacological and non-pharmacological) available for handling this situation in prostate cancer patients receiving ADT.

\section{CLINICAL IMPACT OF BONE MASS LOSS}

A bone fracture in an oncology patient represents a stressful situation that can lead to pain, a need for surgery or radiation therapy, bed rest and a general clear and abrupt decline in quality of life. Not all fractures have the same impact, which depends on the fracture location. Although fractures of the distal skeleton have a small impact and a low risk of associated mortality, multiple vertebral fractures can lead to restrictive respiratory failure and severe kyphosis. Sometimes, the event can be catastrophic for the patient and his or her caregivers, such as with hip fracture, which is associated with a significant mortality risk in male patients over 60 years with or without prostate cancer, as reported in a study based on nearly 4000 patients. ${ }^{10}$ This study ${ }^{10}$ and another study showed that mortality rates because of hip fracture were significantly higher in men than in women. ${ }^{10,11}$ Moreover, the occurrence of a bone fracture at any site in patients with prostate cancer over the course of ADT is associated with a statistically significant decrease in overall survival. ${ }^{12}$ Thus, prevention of bone fractures in these patients is important because it has an impact on overall survival.

The risk of new osteoporotic fractures during ADT increases with the duration of treatment. This phenomenon was highlighted by Daniell in his study published in 1997, which showed that the cumulative incidence of fracture reached almost 50\% 9 years after an orchiectomy was performed. ${ }^{5}$ Other clinical study $^{13}$ and three large population-based studies have more clearly confirmed the increased risk of osteoporotic fracture in patients during $\mathrm{ADT}^{14-16}$ and its relation to the number of luteinizing hormone-releasing hormone analogue doses received during the first 12 months of treatment. ${ }^{14,16}$

\section{Bone mass loss and fracture risk quantification}

Osteoporosis is a bone metabolic disorder characterized by the loss of bone mass and micro-architectural deterioration of bone tissue. Dual energy X-ray absorptiometry (DXA) is the method of choice for quantifying bone loss. It was first recommended in 1994 by the Food and Drug Administration of the United States as the best method for this determination. Bone mass measurement provides two scores: the $T$ score, which quantifies the loss of bone mass in terms of standard deviations in relation to the normal estimated mass for a young adult; and the $Z$-score, which compares the result with the estimated normal mass for a person of the same age, sex and race. The $Z$-score is used in clinical practice to quantify the relative risk of fracture. According to $T$-score results, the World Health Organization (WHO $)^{17}$ developed a qualitative classification system comprising four groups (Table 1).

It is important to note that in the case of patients with bone metastatic prostate cancer, the bone location where the DXA measurement is conducted must be free of metastatic involvement. The International Society for Clinical Densitometry recommends that bone mass measurements should be performed at the femoral neck and lumbar vertebrae and, only in selected cases, at the distal radius. However, the latter was found to be the most sensitive location for diagnosing osteoporosis in a recent study of prostate cancer patients by our group. ${ }^{18}$ In this study, osteoporosis was diagnosed in 30\% of patients when the bone mass measurement was performed at the distal radius, whereas in the same patients, measurements in lumbar vertebrae or the femoral neck diagnosed osteoporosis in $20 \%$ and $23 \%$ of the patients, respectively. In addition, patients with prostate cancer are generally of advanced age and may have vertebral osteophytosis, aortic calcifications or degenerative pelvic bone problems. For all these reasons, we recommend estimating BMD in prostate cancer patients at the distal radius in addition to the locations recommended by the WHO (lumbar spine and femoral neck).

Table 1 World Health Organization (WHO) definition of osteoporosis and osteopaenia according to $T$-score

\begin{tabular}{ll}
\hline Stage & T-score value \\
\hline Normal & $>-1$ \\
Osteopaenia & {$[-1,-2.5]$} \\
Osteoporosis & $<-2.5$ \\
Severe osteoporosis & $<-2.5+$ fragility fracture
\end{tabular}




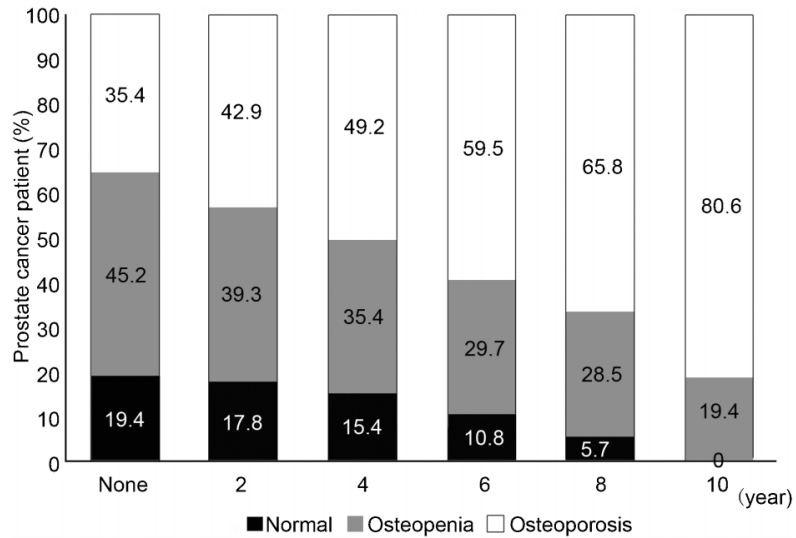

Figure 1 Progressive bone mass loss in prostate cancer patients treated with androgen-deprivation therapy.

The WHO recently proposed a new instrument for the assessment of fracture risk. It is called FRAX, and it calculates the patient's osteoporotic fracture risk based on a series of clinical and epidemiological characteristics (age, sex, body mass index, smoking habit and others). FRAX was used in a cohort of 363 prostate cancer patients receiving ADT to estimate the risk of fracture. The results show that, without using DXA data, age is a crucial predictor that discriminates between patients with a high and low risk of fracture (with a cutoff at 70 years of age), and the incorporation of DXA adjusted the fracture risk estimation to a lower age. ${ }^{19}$

\section{Bone mass loss characteristics during ADT}

In men, age is associated with decreases in testosterone and oestradiol levels, resulting in an annual decrease in bone mass of approximately $1 \%$. In our experience, ${ }^{13}$ before starting ADT, about $15 \%$ and $30 \%$ of male patients had osteopaenia and osteoporosis, respectively. Another study ${ }^{20}$ showed that the percentage of patients with osteoporosis before beginning ADT was $42 \%$. Thus, a significant percentage of prostate cancer patients have a pre-existing low BMD before starting ADT. Once ADT has been initiated, the risk of hip fracture increases progressively from the first year, reaching a relative risk of $4 \%$ in those patients who have received ADT for more than 5 years. ${ }^{13}$ The addition of bicalutamide to castration in order to achieve maximum androgen blockage does not alter the incidence of osteoporosis. ${ }^{13}$ Over the course of ADT, the bone mass loss in patients with prostate cancer is higher than that observed in female breast cancer patients treated with aromatase inhibitors who are either in natural menopause or postmenopausal. ${ }^{21}$

As previously mentioned, bone mass loss during ADT can begin in the first year. ${ }^{22}$ Our group showed that bone loss is most pronounced during the first year of treatment but is subsequently maintained at a slower pace. ${ }^{23}$ Beyond the second year, ${ }^{24}$ we reported a progressive decrease in bone mass over the entire duration of treatment. We observed an osteoporosis rate of $80 \%$ after 10 years, and no patients had a BMD within the normal range (Figure 1).

\section{PREVENTION AND TREATMENT OF BONE MASS LOSS}

Given the evidence demonstrating a direct relationship between ADT and decreased BMD with an increased risk of osteoporotic fractures, it is imperative for clinicians to be aware of preventive measures, which are discussed below. For preventing a reduction in BMD, we should highlight a number of avoidable factors for patients treated with ADT. Patients should be advised to abstain from smoking and to maintain moderate alcohol consumption. Although the exact mechanism is not clearly understood, cigarette smoking is a risk factor for osteoporosis. ${ }^{25}$
On the other hand, moderate alcohol intake is associated with lower risk of fracture. However, excessive alcohol consumption is associated with low BMD and increased fracture risk among men and women. ${ }^{26}$ In addition, it is estimated that the optimal dietary calcium for middleaged men ( $<65$ years) and women (premenopausal) is $1000 \mathrm{mg} \mathrm{day}^{-1}$ or, for older individuals, $1200-1500 \mathrm{mg}$ day ${ }^{-1} \cdot{ }^{27}$ Moreover, a study showed that $93 \%$ of prostate cancer patients treated with ADT had a daily calcium intake below $1000 \mathrm{mg}$, which is less than the level recommended by the WHO for the general population $\left(1200 \mathrm{mg} \mathrm{day}^{-1}\right)$, and that low daily calcium intake was an independent risk factor for osteoporosis development in these patients. ${ }^{28}$ Calcium and vitamin D intake do not have independent preventive effects on the onset of osteoporosis, but their deficit is a major risk factor for this disorder. Finally, we recommend weight-bearing exercise (one of the most important bone-forming stimuli) for patients treated with ADT. Specifically vigorous weight-bearing exercise, such as running and weight training, have been shown actually to increase BMD, whereas less vigorous weight-bearing exercise, such as walking or low-impact aerobics, only protects against further bone loss. ${ }^{29}$

Several studies have evaluated different ADT administration strategies for improving bone mass preservation; among these strategies, delaying the start of hormone therapy was studied. This strategy does not change the survival of metastatic patients if ADT is initiated at the time of symptom onset, nor does it decrease survival in a selected group of patients with biochemical recurrence after radical prostatectomy. Intermittent $\mathrm{ADT}$ is another hormonal treatment strategy designed to reduce the adverse effects of hormone therapy. A small study involving 19 patients with metastatic prostate cancer showed that an off-treatment period followed by subsequent ADT reintroduction when prostatespecific antigen levels reached a predetermined threshold had a favourable effect on bone mass compared with the results obtained in patients on continuous $\mathrm{ADT}^{30}{ }^{30}$ In a small randomized study, bicalutamide monotherapy at a dose of $150 \mathrm{mg}$ day $^{-1}$ resulted in patients experiencing a small increase in $\mathrm{BMD},{ }^{31}$ and it could therefore be considered an option for management of these patients. However, the efficacy and safety of both strategies (intermittent ADT and bicalutamide monotherapy) should be confirmed in future studies with larger sample sizes. Thus, continuous ADT remains the current standard hormonal treatment for prostate cancer, with the possibility of altering the treatment initiation time in selected patients.

In randomized placebo-controlled trials, bisphosphonates (alendronate and zoledronic acid) and the anti-RANKL antibody denosumab have shown beneficial effects in preserving bone mass in patients undergoing ADT (Table 2). Bisphosphonates and denosumab (a completely human monoclonal antibody that specifically binds to RANKL, a mediator of osteoclast activity) suppress osteoclast activity by inhibiting the development, survival and activation of osteoclasts.

Another drug, Toremifene (a selective estrogen receptor modulator) also proved, in a double-blind placebo-controlled phase III study, its capacity to improve BMD as well as to decrease the incidence of new vertebral fractures in men receiving ADT for prostate cancer. ${ }^{32}$

\section{Studies evaluating bisphosphonates}

Bisphosphonates have shown some activity for the preservation of BMD during ADT. Indeed, the earliest bisphosphonate used in clinical practice, etidronate, was tested in this setting and reduced but did not completely prevent bone loss in men who developed hypogonadism. ${ }^{33}$

A Japanese study reported by Izumi et al. ${ }^{34}$ showed that risedronate recovers bone loss in prostate cancer patients treated with ADT. This was a prospective observational study in which 60 patients with 
Table 2 Randomized clinical trials evaluating different treatments in order to prevent bone mass loss in prostate cancer patients treated with androgen-deprivation therapy

\begin{tabular}{|c|c|c|c|c|c|}
\hline Author & $\mathrm{n}$ & Study design & Treatment length & Variables & Results \\
\hline Smith et al. ${ }^{36}$ & 106 & $\begin{array}{l}\text { Zoledronic acid iv ( } 4 \text { mg } \\
\text { per } 3 \text { month) vs. placebo }\end{array}$ & 1 year & BMD \% change at lumbar spine & $\begin{array}{l}+5.6 \% \text { zoledronic acid vs. }-2.2 \% \\
\quad \text { placebo }\end{array}$ \\
\hline Michaelson et al. ${ }^{37}$ & 40 & $\begin{array}{l}\text { Zoledronic iv ( } 4 \mathrm{mg} \mathrm{day}^{-1} \text { ) } \\
\text { vs. placebo }\end{array}$ & 1 year & BMD \% change at lumbar spine & $\begin{array}{l}+4.0 \% \text { zoledronic acid vs. }-3.1 \% \\
\text { placebo }\end{array}$ \\
\hline Greenspan et al. ${ }^{38}$ & 112 & $\begin{array}{l}\text { Oral alendronate } \\
\qquad\left(70 \mathrm{mg} \mathrm{week}^{-1}\right) \text { vs. placebo }\end{array}$ & 1 year & BMD \% change at lumbar spine & $\begin{array}{l}+3.7 \text { alendronate vs. }-1.4 \% \\
\text { placebo }\end{array}$ \\
\hline Smith et al. ${ }^{39}$ & 748 & $\begin{array}{l}\text { Denosumab sc ( } 60 \mathrm{mg} \\
\text { per } 6 \text { month) vs. placebo }\end{array}$ & 3 years & $\begin{array}{l}\text { BMD \% change at lumbar spine and } \\
\text { vertebral fractures }\end{array}$ & $\begin{array}{l}+5.5 \% \text { denosumab vs. }-1 \% \\
\text { placebo ( } 24 \text { months); } 1.5 \% \\
\text { denosumab vs. } 3.9 \% \text { placebo ( } 36 \\
\text { months) }\end{array}$ \\
\hline
\end{tabular}

Abbreviations: BMD, bone mineral density; iv, intravenous; sc, subcutaneous.

prostate cancer receiving ADT were enrolled. The autors analysed 29 and 27 patients in the risedronate and control groups, respectively. The lumbar spine BMD was determined by DXA. Taking into account that the initial mean BMD was significantly lower in the risedronate group, the study showed that the mean percentages of changes in the $\mathrm{BMD} /$ young adult mean ratio of the risedronate group and control group were $+2.6 \% \pm 4.5 \%$ and $-2.8 \% \pm 2.6 \%$ after 1 year, respectively $(P=0.0001)$, so oral administration of risedronate is effective for the recovery of $\mathrm{ADT}$-induced bone loss in patients with prostate cancer.

Moreover, Smith et al. ${ }^{35}$ reported the results of a randomized controlled study to prevent osteoporosis during ADT for prostate cancer. Forty-seven men with locally advanced or recurrent prostate cancer and no bone metastases were randomly assigned to receive leuprolide alone or leuprolide and pamidronate $60 \mathrm{mg}$ intravenously every 12 weeks. BMDs of the posteroanterior lumbar spine and proximal femur were measured by DXA. In men treated with leuprolide alone, BMD decreased by $3.3 \% \pm 0.7 \%$ in the posteroanterior lumbar spine and by $1.8 \% \pm 0.4 \%$ in total hip, while mean BMD did not change significantly at any skeletal site in men treated with both leuprolide and pamidronate. The authors concluded that pamidronate prevents bone loss in the hip and lumbar spine during ADT.

Among the studies on bisphosphonates, we would like to highlight three randomized placebo-controlled clinical trials: two with zoledronic acid $^{36,37}$ and one with alendronate. ${ }^{38}$ The primary endpoint of these studies was to evaluate BMD variation after 1 year of ADT.

In 2003, Smith et al. ${ }^{36}$ randomized 106 patients with stage M0 (no distant metastases) prostate cancer who were beginning initial ADT, to receive either $4 \mathrm{mg}$ zoledronic acid or placebo every 3 months for 1 year. After randomisation, 55 subjects received zoledronic acid while 51 subjects received placebo. Study drug was administered in a double-blind fashion. All subjects were instructed to take a calcium supplement (500 mg) and multivitamin containing vitamin D (400 IU) daily. The primary efficacy variable was the percent change from baseline to the end of the study in BMD of lumbar vertebrae L2-L4 as mesured by DXA. $\mathrm{BMD}$ of other skeletal locations was also measured. Lumbar spine BMD increased $5.6 \%$ from baseline to 1 year in the zoledronic acid group $(P<0.001)$ and decreased $2.2 \%$ in the placebo group. The difference of $7.8 \%$ (95\% confidence interval (CI): $5.6 \%-10.0 \%$ ) in mean percent change at 1 year between the two groups was statistically significant $(P<0.001)$. In the zoledronic acid group, BMD also increased significantly from baseline in the femoral neck, trochanter and total hip. The mean difference in percent change from baseline differed significantly between zoledronic acid and placebo in the femoral neck, trochanter and total hip $(P<0.001)$ but not in the non-dominant forearm. No significant differences regarding adverse events were reported.
Four years later, Michaelson et al. ${ }^{37}$ evaluated the efficacy of annual zoledronic acid in men receiving ADT for prostate cancer. All participants had non-metastatic and non-progressive prostate cancer treated with a GnRH agonist at study entry. Forty-four eligible patients were randomly assigned to receive zoledronic acid $4 \mathrm{mg}$ $(n=22)$ or placebo $(n=22)$ intravenously on day one only. All patients received calcium carbonate (500 $\mathrm{mg}$ daily) and a daily multivitamin containing vitamin D (400 IU). The pimary study endpoint was the percent change in the BMD of the posteroanterior lumbar spine from baseline to month 12. Mean percent changes in BMD of the posteroanterior lumbar spine differed significantly between groups. Mean ( \pm s.e.) $\mathrm{BMD}$ of the posteroanterior lumbar spine decreased by $3.1 \% \pm 1.0 \%$ in the placebo group from baseline to 12 months. In contrast, BMD increased by $4.0 \% \pm 1.0 \%$ in the zoledronic acid group $(P<0.001)$. The between-group difference in percent change from baseline to 12 months was 7.1\% (95\% CI: 4.2\%-10.0\%). Mean percent changes in BMD of the total hip and trochanter also differed significantly between groups. The between-group differences in percent change from baseline to 12 months were $2.6 \%$ (95\% CI: $0.8 \%$ $4.2 \%$ ) for the total hip, $3.1 \%$ (95\% CI: $0.9 \%-5.3 \%$ ) for the trochanter and $4.4 \%$ (95\% CI: $-0.1 \%-4.4 \%)$ for the femoral neck. No serious adverse events related to treatment in either group were reported.

In 2007, Greenspan et al. ${ }^{38}$ sought to determine whether once-weekly alendronate would improve bone mass and reduce bone turnover in men with non-metastatic prostate cancer receiving ADT. Patients recruited had just initiated ADT (within the previous 6 months) or were receiving long-term ADT. One hundred and twelve patients were randomly assigned to receive alendronate $(n=56), 70 \mathrm{mg}$ once weekly, or matching placebo $(n=56)$. Calcium and vitamin D supplements $(500 \mathrm{mg}$ of calcium and 200 IU of vitamin D per tablet) were provided to ensure that patients' calcium intake was greater than $1000 \mathrm{mg}$ day $^{-1}$. The primary outcome was the percentage change in posterior-anterior spine BMD at 12 months. Secondary outcomes included percentage changes at the total hip and femoral neck at 12 months. The estimated BMD change for men in the placebo group after 1 year was $-1.4 \%$ (95\% CI: $-2.7 \%$ to $-0.03 \% ; P=0.045)$ at the postero-anterior spine. By comparison, the estimated BMD change after 1 year in men who received alendronate treatment increased by $3.7 \%$ (95\% CI: $2.8 \%-4.6 \%$; $P<0.001$ ). The estimated changes in BMD were significantly different between groups. In percentage points, these changes were 5.1\% (95\% CI: 3.5\%-6.7\%; $P<0.001)$ at the spine, $1.4 \%$ (95\% CI: $0.5 \%-2.4 \% ; P=0.002)$ at the total hip and 2.3\% (95\% CI: $1.0 \%-3.7 \% ; P<0.001)$ at the femoral neck. The two groups did not statistically differ in adverse events.

As we can see, the three trials showed an increase in BMD in the active treatment group, whereas a decrease in BMD was reported in the placebo 
group. However, none of these studies showed any change in osteoporotic fracture risk. Moreover, the choice of drug, route of administration (oral or intravenous) and optimal treatment duration are still unresolved issues of bisphosphonate therapy in patients treated with ADT.

\section{Denosumab}

In 2009, the results of a randomized double-blind study comparing denosumab with placebo in patients with metastatic prostate cancer were published. ${ }^{39}$ This study randomly assigned 1468 patients to subcutaneously receive the placebo or denosumab at a dose of $60 \mathrm{mg}$ per 6 months. The primary endpoint of this study was the percent change in BMD at the lumbar spine after 24 months, but in contrast to the bisphosphonate studies, this study included the incidence of new vertebral fractures as a key secondary endpoint. The results of this study showed that compared with placebo, denosumab therapy was associated with significant increases in BMD. Significant differences between the two groups were seen as early as 1 month. Moreover, patients treated with denosumab had a significantly lower rate of new vertebral fractures at 12, 24 and 36 months. At 36 months, the incidence of new vertebral fractures was $1.5 \%$ in patients treated with denosumab and $3.9 \%$ in patients treated with the placebo, with a $62 \%$ relative reduction in vertebral risk fracture $(P<0.006)$. The rates of adverse events were similar between the two groups. It is noteworthy that no cases of osteonecrosis of the jaw or delayed healing of fractures were reported.

\section{Toremifene}

As we mentioned before, the intended therapeutic effect of ADT is to reduce testosterone to castrate levels. Because estradiol is derived from the peripheral conversion of testosterone by aromatase, ADT also markedly decreases serum estradiol levels, which may result in unintended estrogen deficiency side effects. Estradiol is critical to bone formation and bone resorption in men. ${ }^{40}$

Toremifene is a second-generation selective estrogen receptor modulator. Smith et al. ${ }^{32}$ conducted a large randomized placebocontrolled trial evaluating whether toremifene could decrease the incidence of new vertebral fractures in men on ADT. Secondary efficacy endpoints included incidence of fragility fractures; BMD at the spine, hip and femoral neck; as well as changes in bone turnover markers and changes in serum lipids. The study included 1284 subjects who were receiving ADT for histologically documented prostate cancer and who were at increased risk for fracture based on age 70 years or older, or osteopaenia at the femoral neck or L1-L4 lumbar spine. Six hundred and forty-six subjects were assigned to toremifene $80 \mathrm{mg}$ by mouth daily and 638 to placebo. Compared with placebo, BMD increased significantly at the lumbar spine $(2.3 \%, 95 \%$ CI: $1.6-3.1)$, total hip (1.9\%, 95\% CI: 1.3-2.4) and femoral neck (1.9\%, 95\% CI: $1.2-2.7)$ in the toremifene group ( $P<0.0001$ for each comparison). Moreover, the 2-year incidence of new vertebral fractures was $4.9 \%$ (23 patients) with placebo vs. $2.5 \%$ (11 patients) with toremifene, a significant relative risk reduction of $50 \%$ ( $95 \%$ CI: $-1.5 \%$ to $-75.0 \%$; $P<0.05$ ). The incidence of all fractures was $10.1 \%$ (47 patients) with placebo and $6.3 \%$ with toremifene (28 patients), a significant relative risk reduction of $38 \%$ ( $95 \% \mathrm{CI}: 2.2 \%-60.2 \%$; $P=0.036$ ). Although toremifene was well tolerated overall, an increased rate of venous thromboembolic events (VTEs) was observed, primarily in men with known risk factors, including age 80 years or greater. VTEs were reported in 17 subjects $(2.6 \%)$ in the toremifene group and seven $(1.1 \%)$ in the placebo group.

Because of these results, Smith et al. ${ }^{4}$ performed a post hoc analysis of the phase III study to assess the efficacy and safety of toremifene $80 \mathrm{mg}$ in men younger than 80 years. This post hoc analysis included 847 men younger than 80 years, of whom 430 received toremifene $80 \mathrm{mg}$ by mouth daily and 417 received placebo. Baseline characteristics were similar between the treatment groups. The percent increase in BMD from baseline to month 24 was significantly greater for toremifene than for placebo at all measured sites in subjects younger than 80 years. As in the previous study, the 2-year incidence of new vertebral fractures was $1 \%$ in the toremifene group $v s .4 .8 \%$ in the placebo group $(P<0.005)$, corresponding to a $79.5 \%$ relative risk reduction (95\% CI: $29.8 \%-$ $94.0 \%$ ). Compared with placebo, toremifene significantly decreased the incidence of non-traumatic fractures or greater than $7 \%$ bone loss by month 24 . This was a $56.2 \%$ relative risk reduction (95\% CI: $34.4 \%-$ $70.8 \%)$. In men younger than 80 years, toremifene was associated with a slight, nonsignificant increase in the VTE rate compared with placebo $(2.1 \%$ vs. $1.0 \%$; $P=0.26)$. Specifically nine of $430 \mathrm{men}$ in the toremifene group had a VTE $v s$. four of 417 in the placebo group.

\section{DIAGNOSTIC AND PREVENTIVE RECOMMENDATIONS}

The National Osteoporosis Foundation $(\mathrm{NOF})^{41}$ recently published a list of recommendations for osteoporotic patients (Table 3). In summary, the NOF recommends the testing of BMD for all patients who have any of the risk factors. It is remarkable that all men aged 70 years or older are included in this group. Regarding treatment, the NOF recommends calcium (1200 mg or more daily) and vitamin D (800-1000 IU daily) for all adults, and it recommends treatment consideration for patients aged 50 years or older with a history of hip or vertebral fracture, osteoporosis at the femoral neck or spine, as well as osteopaenia at the femoral neck or spine with a high probability of future fracture as determined by the FRAX algorithm. Similarly, the latest guidelines of the European Association of Urology, published in 2010, also recommend evaluating BMD by DXA in patients receiving $\mathrm{ADT}^{42}$

\section{TREATMENT RECOMMENDATIONS}

The very high rate of bone loss and the high incidence of fractures indicate that patients at risk of cancer treatment-induced bone loss need to be carefully monitored and stratified for fracture risk.

Although there is a strong evidence of efficacy in prevention of bone loss and reduction in fracture risk for many drugs approved for male osteoporosis and postmenopausal osteoporosis, for cancer treatmentinduced bone loss, there were actually no drugs approved for this indication. This situation changed on 16 September 2011 when the Food and Drug Administration granted approval for Denosumab (Prolia, made by Amgen, Inc., Thousand Oaks, CA, USA) as a treatment to increase bone mass in patients who are at high risk of fracture from receiving $\mathrm{ADT}$ for non-metastatic prostate cancer.

\section{CONCLUSIONS}

Osteoporosis is a frequent clinical problem with significant associated morbidity and mortality. ADT, which represents the first-line therapy for most advanced and metastatic prostate cancer patients, is related to progressive bone mass loss that can lead to osteoporosis. Thus, as urologists, we should be aware of the bone health of our prostate cancer patients who are receiving $\mathrm{ADT}$ in order to diagnose bone problems early and begin general measures and/or indicated treatments when necessary.

\section{COMPETING FINANCIAL INTERESTS}

The authors have nothing to disclose and have no conflicts of interest pertaining to the article. This includes any commercial interests that may be construed to have influenced opinion such as research 


\section{Table 3 Relevant NOF recommendations}

\section{Screening:}

NOF recommends testing of bone mineral density for:

- all men aged 70 years or older

- adults who have a fracture after age 50

- adults with a condition or taking a medicine associated with low bone mass or bone loss

- anyone considered for pharmacological therapy for osteoporosis

- anyone being treated for osteoporosis, to monitor treatment effect

- anyone not receiving therapy in whom evidence of bone loss would lead to treatment

Treatment:

NOF recommends calcium (1200 mg or greater daily) and vitamin D (800-1000 IU daily) for all and consideration for treatment if age 50 or older and any of the following:

- history of hip or vertebral fracture

- $T$-score 2.5 or less at the femoral neck or spine (secondary causes evaluated)

- low $T$-score at femoral neck or spine $(-1.0$ to -2.5$)$ and either of the following:

10-year probability of a hip fracture 3\% or greater by the United States adapted WHO/FRAX algorithm

10-year probability of a major osteoporosis-related fracture $20 \%$ or greater by the United States adapted WHO/FRAX algorithm

Abbreviations: NOF, National Osteoporosis Foundation; WHO, World Health Organization.

Adapted from National Osteoporosis Fundation's Clinician's Guide to Prevention and Treatment of Osteoporosis. ${ }^{41}$

funding, consultancy services, serving on speakers' bureaus, travel expenses, appearance fees, the receipt of share options or direct employment by a commercial entity.

1 Jemal A, Siegel R, Xu J, Ward E. Cancer statistics, 2010. CA Cancer J Clin 2010; 60 277-300.

2 Yoneda T, Hiraga T. Crosstalk between cancer cells and bone microenvironment in bone metastasis. Biochem Biophys Res Commun 2005; 328: 679-87.

3 [No authors listed.] Medicine 2006; 9 Extr. 1:1-7.

4 Smith MR, Malkowicz SB, Brawer MK, Hancock ML, Morton RA et al. Toremifene decreases vertebral fractures in men younger than 80 years receiving androgen deprivation therapy for prostate cancer. J Urol 2011; 186: 2239-44.

5 Daniell HW. Osteoporosis after orchiectomy for prostate cancer. J Urol 1997; 157: 439-44.

6 Townsend MF, Sanders WH, Northway RO, Graham SD. Bone fractures associated with luteinizing hormone-releasing hormone agonists used in the treatment of prostate carcinoma. Cancer 1997; 79: 545-50.

7 Shahinian VB, Kuo YF, Freeman JL, Orihuela E, Goodwin JS. Increasing use of gonadotropin-releasing hormone agonists for the treatment of localized prostate carcinoma. Cancer 2005; 103: 1615-24.

8 Barry MJ, Delorenzo MA, Walker-Corkery ES, Lucas FL, Wennberg DC. The rising prevalence of androgen deprivation among older American men since the advent of prostate-specific antigen testing: a population-based cohort study. BJU Int 2006; 98: 973-8.

9 Kong YY, Yoshida H, Sarosi I, Tan HL, Timms E et al. OPGL is a key regulator of osteoclastogenesis, lymphocyte development and lymph-node organogenesis. Nature 1999; 397: 315-23.

10 Jiang HX, Majumdar SR, Dick DA, Moreau M, Raso J et al. Development and initial validation of a risk score for predicting in-hospital and 1 -year mortality in patients with hip fractures. J Bone Miner Res 2005; 20: 494-500.

11 Roche JJ, Wenn RT, Sahota O, Moran CG. Effect of comorbidities and postoperative complications on mortality after hip fracture in elderly people: prospective observational cohort study. BMJ 2005; 331: 1374.

12 Oefelein MG, Richiutti V, Conrad W, Resnick MI. Skeletal fractures negatively correlate with overall survival in men with prostate cancer. J Urol 2002; 168: 1005-7.

13 Morote J, Martinez E, Trilla E, Esquena S, Abascal JM et al. Osteoporosis during continuous androgen deprivation: influence of the modality and length of treatment. Eur Urol 2003; 44: 661-5.

14 Dickman PW, Adolfsson J, Astrom K, Steineck G. Hip fractures in men with prostate cancer treated with orchiectomy. J Urol 2004; 172: 2208-12.

15 Smith MR, Lee WC, Brandman J, Wang Q, Botteman M et al. Gonadotropin-releasing hormone agonists and fracture risk: a claims-based cohort study of men with nonmetastatic prostate cancer. J Clin Oncol 2005; 23: 7897-903.

16 Shahinian VB, Kuo YF, Freeman JL, Goodwin JS. Risk of fracture after androgen deprivation for prostate cancer. N Engl J Med 2005; 352: 154-64.

17 Assesment of fracture risk and its application to screening for postmenopausal osteoporosis. Report of a WHO Study Group. World Health Organ Tech Rep Ser 1994; 843: 1-129.

18 Morote J, Planas J, Mir MC, Raventos CX, Encabo G et al. 33\% radius evaluation to assess bone mineral density in prostate cancer patients. World J Urol 2010; 29: 815-9.

19 Saylor PJ, Kaufman DS, Michaelson MD, Lee RJ, Smith MR. Application of a fracture risk algorithm to men treated with androgen deprivation therapy for prostate cancer. J Urol 2010; 183: 2200-5.

20 Hussain SA, Weston R, Stephenson RN, George E, Parr NJ. Immediate dual energy Xray absorptiometry reveals a high incidence of osteoporosis in patients with advanced prostate cancer before hormonal manipulation. BJU Int 2003; 92: 690-4.
21 Higano CS. Androgen-deprivation-therapy-induced fractures in men with nonmetastatic prostate cancer: what do we really know? Nat Clin Pract Urol 2008; 5: 24-34.

22 Greenspan SL, Coates P, Sereika SM, Nelson JB, Trump DL et al. Bone loss after initiation of androgen deprivation therapy in patients with prostate cancer. J Clin Endocrinol Metab 2005; 90: 6410-7.

23 Morote J, Orsola A, Abascal JM, Planas J, Trilla E et al. Bone mineral density changes in patients with prostate cancer during the first 2 years of androgen suppression. $J$ Urol 2006; 175: 1679-83; discussion 1683.

24 Morote J, Morin JP, Orsola A, Planas J, Trilla E et al. Prevalence of osteoporosis during long termandrogen deprivation therapy in patients with prostatecancer. Urology2007;69:500-4

25 Vogel JM, Davis JW, Nomura A, Wasnich RD, Ross PD. The effects of smoking on bone mass and the rates of bone loss among elderly Japanese-American men. $J$ Bone Miner Res 1997; 12: 1495-501.

26 Ross PD. Risk factors for osteoporotic fracture. Endocrinol Metab Clin North Am 1998; 27: 289-301.

27 NIH consensus conference. Optimal calclium intake. JAMA 1994; 272: 1942-8.

28 Planas J, Morote J, Orsola A, Salvador C, Trilla E et al. The relationship between daily calcium intake and bone mineral density in men with prostate cancer. BJU Int 2007; 99: 812-5; discussion 815-6.

29 Todd JA, Robinson RJ. Osteoporosis and exercise. Postgrad Med J 2003; 79: 320.

30 Higano C, Shields A, Wood N, Brown J, Tangen C. Bone mineral density in patients with prostate cancer without bone metastases treated with intermittent androgen suppression. Urology 2004; 64: 1182-6.

31 Smith MR, Goode M, Zietman AL, McGovern FJ, Lee $\mathrm{H}$ et al. Bicalutamide monotherapy versus leuprolide monotherapy for prostate cancer: effects on bone mineral density and body composition. J Clin Oncol 2004; 22: 2546-53.

32 Smith MR, Morton RA, Barnette KG, Sieber PR, Malkowicz SB et al. Toremifene to reduce fracture risk in men receiving androgen deprivation therapy for prostate cancer. J Urol 2010; 184: 1316-21.

33 Diamond T, Campbell J, Bryant C, Lynch W. The effect of combined androgen blockade on bone turnover and bone mineral densities in men treated for prostate carcinoma: longitudinal evaluation and response to intermittent cyclic etidronate therapy. Cancer 1998; 83: 1561-6.

34 Izumi K, Mizokami A, Sugimoto K, Narimoto K, Miwa S et al. Risedronate recovers bone loss in patients with prostate cancer undergoing androgen-deprivation therapy. Urology 2009; 73: 1342-6.

35 Smith MR, Mc Govern FJ, Zietman AL, Fallon MA, Hayden DL et al. Pamidronate to prevent bone loss in men receiving gonadotropin releasing hormone agonist therapy for prostate cancer. N Engl J Med 2001; 345: 948-55.

36 Smith MR, Eastham J, Gleason DM, Shasha D, Tchekmedyian S et al. Randomized controlled trial of zoledronic acid to prevent bone loss in men receiving androgen deprivation therapy for nonmetastatic prostate cancer. J Urol 2003; 169: 2008-12.

37 Michaelson MD, Kaufman DS, Lee H, McGovern FJ, Kantoff PW et al. Randomized controlled trial of annual zoledronic acid to prevent gonadotropin-releasing hormone agonist-induced bone loss in men with prostate cancer. J Clin Oncol 2007; 25: 1038-42.

38 Greenspan SL, Nelson JB, Trump DL, Resnick NM. Effect of once-weekly oral alendronate on bone loss in men receiving androgen deprivation therapy for prostate cancer: a randomized trial. Ann Intern Med 2007; 146: 416-24.

39 Smith MR, Egerdie B, Hernandez Toriz N, Feldman R, Tammela TL et al. Denosumab in men receiving androgen-deprivation therapy for prostate cancer. N Engl J Med 2009; 361: 745-55.

40 Falahati-Nini A, Riggs BL, Atkinson EJ, O'Fallon WM, Eastell R et al. Relative contributions of testosterone and estrogen in regulating bone resorption and formation in normal elderly men. J Clin Invest 2000; 106: 1553-60.

41 National Osteoporosis Foundation. Clinician's Guide to Prevention and Treatment of Osteoporosis. Washington, DC: National Osteoporosis Foundation; 2010.

42 Heidenreich A, Bastian PJ, Bellmunt J, Bolla M, Joniau S et al. Guidelines on prostate cancer 2010. Available from: http://uroweb.org/guidelines/online-guidelines/ 